Original Research Paper

\title{
Neuronal Necrosis Due to a Lethal Epileptic Syndrome Affecting FVB Strain $\beta$-Actin Luciferase Transgenic Mice
}

\author{
${ }^{1}$ Ahmed Shoieb, ${ }^{2}$ Rachel Allavena and ${ }^{3}$ Abdelazim Ibrahim \\ ${ }^{I}$ Pfizer Drug Safety Research and Development, Groton, CT 06340, USA \\ ${ }^{2}$ School of Veterinary Science, Faculty of Science, The University of Queensland, Australia \\ ${ }^{3}$ Department of Pathology, Faculty of Veterinary Medicine, Suez Canal University, Ismailia, Egypt
}

Article history

Received: 23-06-2014

Revised: 07-07-2014

Accepted: 24-11-2014

Corresponding Author:

Abdelazim Ibrahim

Department of Pathology,

Faculty of Veterinary

Medicine, Suez Canal

University, Ismailia, Egypt

E-mail: abdelazimibrahhim@hotmail.com

\begin{abstract}
The FVB is the most often inbred mouse strain used in transgenic research. This strain is recognised to be affected with a disease known as FVB Lethal Epileptic Syndrome (FLES). A case series of FVB mouse lethal epileptic syndrome in a cohort of commercially procured $\beta$ actin luciferase transgenic FVB mice was diagnosed based on the clinical signs, post-mortem and histopathologic findings and immunohistochemical analysis of glutamate receptors. Observed clinical signs included nonresponsiveness, hunched posture, piloerection. Histopathologic lesions included moderate to severe acute neuronal necrosis affecting predominantly the cerebral cortex, thalamus and hippocampus with hepatic coagulative necrosis. Glutamate receptors showed mild to marked positive expression within the affected neurons. Glutamate receptor may have a potential role in the pathogenesis of FLES.
\end{abstract}

Keywords: FVB Mice, Lethal Epileptic Syndrome, Neuronal Necrosis, Glutamate Receptors

\section{Introduction}

In the last two decades, transgenic mice have revolutionized medicine biotechnology allowing researcher an opportunity to study and understand functions of gene products. Transgenic technology has a wide variety of applications in the fields of animal genetic improvement, bioreactor, organ transplant as well as they have become a real model for human pathology model (Jones, 2011; Zhen-Hong et al., 2010). The inbred FVB/N mice, created in the early 1970 s, is the most commonly used strain for transgenic research because of its welldefined inbred background, vigorous reproductive performance and consistently large letters. Moreover, its fertilized egg contains large, prominent pronuclei which facilities microinjection of DNA (Taketo et al., 1991; Goelz et al., 1998; Schauwecker, 2003).

FVB Lethal Epileptic Syndrome (FLES) presents a significant confounding factor on transgenic research using this mouse strain. In some colonies, spontaneous mortalities may reach up 20\% (Mahler et al., 1996; Goelz et al., 1998) the molecular basis of FLES, referred to more colloquially as 'Space-cadet' syndrome, has yet to be determined. The neuronal necrosis observed in this syndrome is similar to that observed in cases of status epilepticus in human which is believed to occur through excitotoxicity (Goelz et al., 1998). Excitotoxic neuronal death is caused by elevated levels of excitatory neurotransmitters, particularly glutamate, which interacts with glutamate receptor-associated ion channels resulting in prolonged depolarization of neurons with increased intracellular calcium and finally cell death (Nakanishi, 1994; 1992). Glutamate Receptors (GluRs) are located primarily on the cell membrane of soma. There are two major types of GluRs, ionotropic (iGluRs) and metabotropic (mGluRs). The iGluR family is subdivided into three types: N-Methyl-D-Aspartate (NMDA), $\alpha-$ Amino-3- hydroxy-5-Methyl-4-isoxazole Propionic Acid (AMPA) and Kainate (Ka) receptors (Nakanishi, 1994; 1992; Dingledine et al., 1999; Hollmann and Heinemann, 1994; Asztely and Gustafsson, 1996; Kong et al., 2008).

In the present article, we reported the occurrence of lethal epileptic syndrome in FVB mice with exploring the expression of the glutamate with immunohistochemistry.

\section{Material and Method}

Mice

A colony of mature female transgenic $\beta$-actin luciferase mouse on an FVB strain background were 
kept at Pfizer Global Research and Development (Sandwich, Kent, UK) as stock animals without undergoing any procedure or treatment. They were supplied with ad libitum, Special Diet Services pellets. The room temperature was maintained at $21 \pm 2{ }^{\circ} \mathrm{C}$ and a relative humidity of $55 \pm 10 \%$, with a 12 $\mathrm{h}$ light/dark cycle. The animals were observed for any clinical signs twice daily.

\section{Histopathology and Immunofluorescence}

Clinically affected and dead animals were subjected to a full necropsy. Whole brains and liver, kidney, lung and heart were dissected, fixed in 10\% neutral buffered formalin and then embedded in paraffin blocks. About 4 $\mu \mathrm{m}$ sections were stained with Haematoxylin and eosin (H\&E). Unstained sections were mounted with DAPI and Fluorescence photomicrographs were captured on a Zeiss Axioplan2 fluorescence microscope using the FITC filter (emission wavelength $518 \mathrm{~nm}$ ).

\section{Ingenuity Pathway Analysis (IPA)}

IPA is a powerful bioinformatics tool that permits the rapid dissection and analysis of physiological pathways (as described in the peer-reviewed published literature-base). The Ingenuity Pathway Analysis tool version 8.0 was used (IPA; Ingenuity® Systems, Inc, Redwood City, CA).

\section{Immunohistochemistry (IHC)}

IHC was performed using the avidin-biotinperoxidase method. Rabbit polyclonal anti-Glutamate Receptor 1 (AMPA subtype), anti- GAD65 + GAD67, anti- NMDAR1 and Anti-mGluR (Dako Corporation, Carpinteria, CA) were used. They were diluted 1:2000, 1: 1000, 1: 500 and 1: 500, respectively, in phosphate buffer saline. Slides were incubated with primary antibodies for $24 \mathrm{~h}$. After rinsing, sections were incubated for $1 \mathrm{hr}$ with appropriate secondary antibodies (Vectors Lab., Burlingame, CA).

\section{Results}

Eighteen (18) of 103 animals in were found severely deteriorated within a $48 \mathrm{~h}$ period. Mice were found to be reluctant to move with a hunched back and were subsequently humanly killed by a $\mathrm{CO} 2$ euthanasia. Clinical signs included dehydration and anorexia, a reddish nasal discharge and wet chin and chest. No seizure like activity was observed in affected mice or others in the colony.

No significant macroscopic abnormalities were noted during the necropsy. On histologic examination, brain exhibited multifocal neuronal necrosis Affected neurons were reduced in size, angular with pyknotic nuclei and deeply eosinophilic cytoplasm.
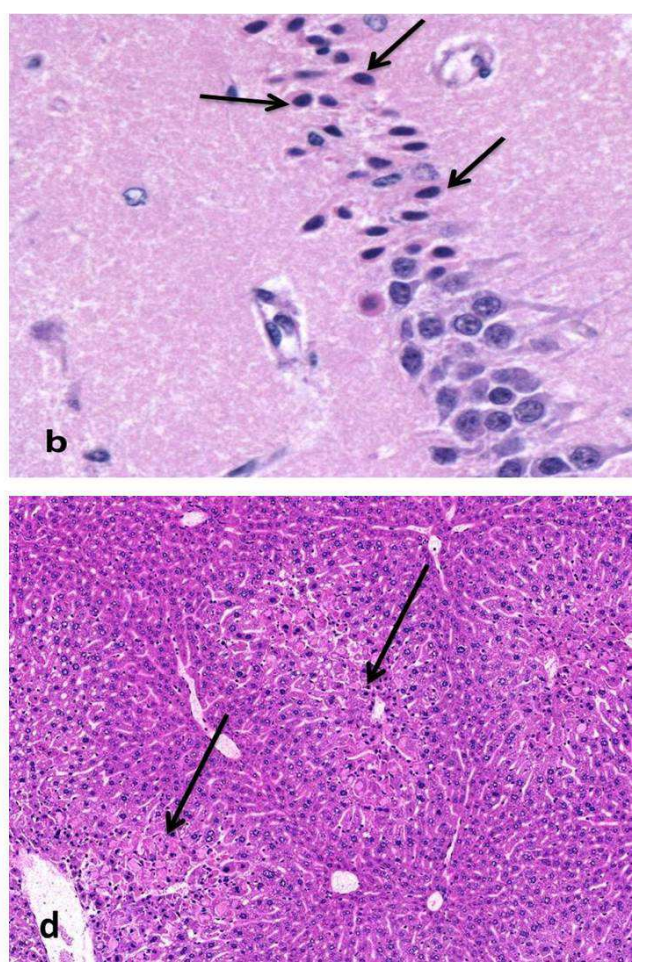

Fig. 1. Lesions in FVB status epilepticus syndrome consist of minimal to marked neuronal necrosis most notable in the hippocampus (a and b) and cortical laminar neurons (c). Sporadic animals show multifocal random acute hepatocellular necrosis (d) attributed to hypoxia during seizuring. Paraffin-embedded Haematoxylin and Eosin stained sections 

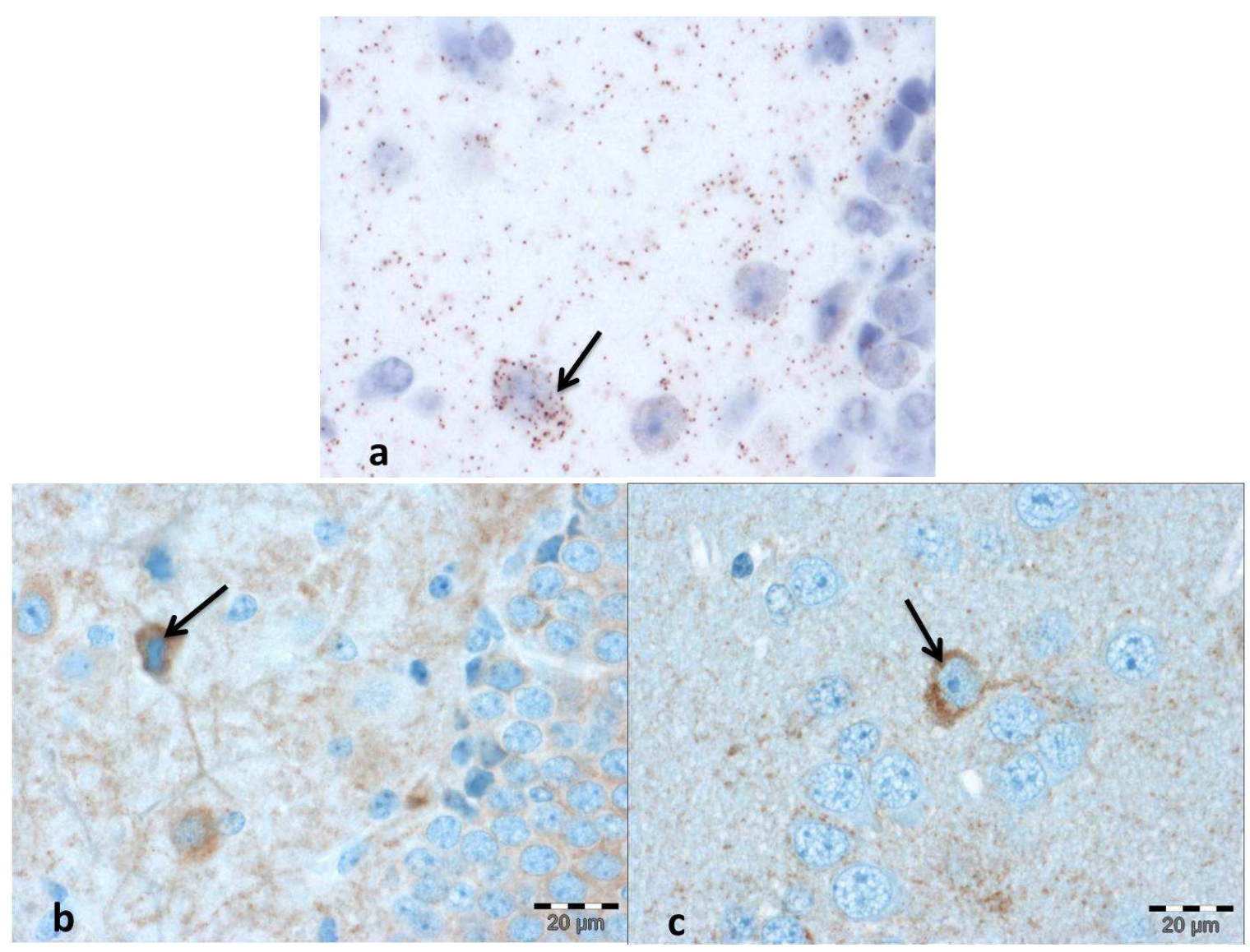

Fig. 2. Immunohistochemical detection of AMPA (a) MGluR1 (b) and GAD (c) receptors protein in the mouse hippocampus showing moderate cytoplasmic expression of these proteins (arrows)

The mostly affected areas were cerebral cortex, thalamus and hippocampus (Fig. 1a-c). Approximately $50 \%$ of animals additionally had multifocal, randomly distributed areas of coagulative necrosis within the liver (Fig. 1d). Necrotic neurons were noted to autofluoresce under the FluorosceinIsothiocyanate (FITC) filter of a fluorescence microscope. The clinical and pathological observations were determined to be consistent with FLES

In order to identify candidate proteins involved in FLES, we used Ingenuity Pathway Analysis (IPA). IPA analysis is commonly used to assess potential downstream implications of target modulation in a toxic setting and support investigative study design.

Immunohistochemical examination for glutamatergic signalling pathway showed various degree of membranous and cytoplasmic expression to AMPA receptor protein in the mouse hippocampus, enhanced by in situ proximity ligase (converts the brown DAB staining into visually discernable dots). Expression is highest in the CA1 subfield in FVB animals compared to $\mathrm{C} 57 / \mathrm{Bl}$ controls. Figure $2 \mathrm{a}$ demonstrates grains over pyramidal neuron layer of CA 1. Furthermore, immunohistochemical detection of MGluR1 receptor protein and GAD in the FVB mouse hippocampus showed strong membranous and cytoplasmic immunoreactivity which is highest in the CA1 subfield (Fig. 2b and c). Conversely, no immunoreactivity staining difference was detected for the NMDA receptor (NMDAR), a glutamate receptor, in FVB animals compared to $\mathrm{C} 57 / \mathrm{Bl}$ controls.

\section{Discussion}

The current paper reports a case series of FVB mouse lethal epileptic syndrome in a cohort of commercially procured $\beta$ actin luciferase transgenic FVB mice. The clinical presentation, associated with brain and liver lesions was classic for that syndrome.

In this study, further investigation of tissue expression of various key proteins in glutamate signal transduction in FVB lethal epileptic syndrome was performed using immunohistochemistry. Glutamate is an amino acid that is considered to be the main excitatory neurotransmittor in mammals. At higher 
concentrations, Glutamate becomes a neurotoxin leading to severe damage and necrosis to the neuron through hyperexictation mechanism (Lipton and Rosenberg, 1994; Meldrum, 1994; Miller et al., 1995; 1997; Ozawa et al., 1998; Schoepp, 1994; Ankarcrona et al., 1995; Lipton and Nicotera, 1998; Rosin et al., 2004; Truong et al., 2008). Immunohistochemical examination showed expression of two key receptors; AMPA receptor protein and MGluR1 receptor protein. No immunoreactivity staining difference was detected for the NMDA Receptor (NMDAR) in FVB animals compared to C57/Bl controls.

Despite the prevalence of FLES in some FVB colonies, seizures are typically rarely observed, probably because seizure activity is more common at night. Goelz et al. (1998) as the underlying cause of FLES is unknown we undertook a preliminary investigation into the role of the glutamatergic pathway in the syndrome.

The neuronal necrosis seen in FLES is similar to that of status epilepticus (Lowenstein and Alldredge, 1998). Increased levels of glutamate, results in the opening ion channels that associated with glutamate receptors, prolonged depolarization of neurons and secondary influx of calcium into the neuron which induce excitotoxic death of the neuron (Schauwecker, 2003). Not only are FVB mice more sensitive to seizure induction; they are also more prone to seizure-associated neuronal cell death once seizures occur, compared to resistant strains like C57Bl/6 (Goelz et al., 1998). Genes may be involved in the process of FLES, particularly those responsible for hippocampal excitability and glutamate release (Nakanishi, 1994).

\section{Conclusion}

Finally, we can conclude that lethal epileptic syndrome hinders the usage of FVB mice for the research purpose, as well as it causes economic loses for transgenic mice industry. Our results highlight the possible role of glutamate in process of that disease, but further investigations are required, like quantitation of glutamate expression using real-time PCR. Moreover, intracellular glutamate metabolising enzymes can be examined.

\section{Acknowledgement}

This work was supported by Pfizer Drug Safety Research and Development.

\section{Funding Information}

Authors are grateful for the support by Pfizer Drug Safety Research and Development.

\section{Author's Contributions}

Authors are equally share in preparing this article.

\section{Ethics}

The authors declared that they had no conflicts of interest with respect to their authorship or the publication of this article.

\section{References}

Ankarcrona, M., J.M. Dypbukt, E. Bonfoco, B. Zhivotovsky and S. Orrenius et al., 1995. Glutamate-induced neuronal death: A succession of necrosis or apoptosis depending on mitochondrial function. Neuron, 15: 961-973.

DOI: $10.1016 / 0896-6273(95) 90186-8$

Asztely, F. and B. Gustafsson, 1996. Ionotropic glutamate receptors. Their possible role in the expression of hippocampal synaptic plasticity. Mol. Neurobiol., 12: 1-11. PMID: 8732537

Dingledine, R., K. Borges, D. Bowie and S.F. Traynelis, 1999. The glutamate receptor ion channels. Pharmacol. Rev., 51: 7-61. PMID: 10049997

Goelz, M.F., J. Mahler, J. Harry, P. Myers and J. Clark et al., 1998. Neuropathologic findings associated with seizures in FVB mice. Lab Anim. Sci., 48: 34-37. PMID: 9517887

Hollmann, M. and S. Heinemann, 1994. Cloned glutamate receptors. Ann. Rev. Neurosci., 17: 31-108. DOI: 10.1146/annurev.ne.17.030194.000335

Jones, D., 2011. Genetic engineering of a mouse: Dr. Frank Ruddle and somatic cell genetics. Yale J. Biol. Med., 84: 117-124.

Kong, S., A. Lorenzana, Q. Deng, T.H. McNeill and P.E. Schauwecker, 2008. Variation in Galr1 expression determines susceptibility to exocitotoxin-induced cell death in mice. Genes Brain Behav., 7: 587-598. DOI: 10.1111/j.1601-183X.2008.00395.X

Lipton, S. and P. Nicotera, 1998. Calcium, free radicals and excitotoxins in neuronal apoptosis. Cell Calcium, 23: 165-171.

DOI: $10.1016 / \mathrm{S} 0143-4160(98) 90115-4$

Lipton, S. and P.A. Rosenberg, 1994. Excitatory amino acids as a final common pathway for neurologic disorders. N. Engl. J. Med., 330: 613-622. DOI: 10.1056/NEJM199403033300907

Lowenstein, D.H. and B.K. Alldredge, 1998. Status epilepticus. N. Engl. J. Med., 338: 970-976. DOI: 10.1056/NEJM199804023381407

Mahler, J., W. Stokes, P.C. Mann, M. Takaoka and R.R. Maronpot, 1996. Spontaneous lesions in aging FVB/N mice. Toxicol. Pathol., 24: 710-716. DOI: $10.1177 / 019262339602400606$

Meldrum, B.S., 1994. The role of glutamate in epilepsy and other CNS disorders. Neurology, 44: S14-23.

Miller, H., A.I. Levey, J.D. Rothstein, A.V. Tzingounis and P.J. Conn, 1997. Alterations in glutamate transporter protein levels in kindling-induced epilepsy. J Neurochem, 68: 1564-1570. DOI: $10.1046 /$ j.1471-4159.1997.68041564.x 
Miller, S., J.P. Kesslak, C. Romano and C.W. Cotman, 1995. Roles of metabotropic glutamate receptors in brain plasticity and pathology. Ann. NY Acad Sci., 757: 460-474.

DOI: $10.1111 / \mathrm{j} .1749-6632.1995 . t b 17506 . x$

Nakanishi, S., 1992. Molecular diversity of glutamate receptors and implications for brain function. Science, 258: 597-603.

DOI: $10.1126 /$ science. 1329206

Nakanishi, S., 1994. Metabotropic glutamate receptors: Synaptic transmission, modulation and plasticity. Neuron, 13: 1031-1037.

DOI: 10.1016/0896-6273(94)90043-4

Ozawa, S., H. Kamiya and K. Tsuzuki, 1998. Glutamate receptors in the mammalian central nervous system. Prog. Neurobiol., 54: 581-618.

DOI: 10.1016/S0301-0082(97)00085-3

Rosin, C., T.E. Bates and S.D. Skaper, 2004. Excitatory amino acid induced oligodendrocyte cell death in vitro: Receptor-dependent and independent mechanisms. J. Neurochem., 90: 1173-1185.

DOI: $10.1111 / \mathrm{j} .1471-4159.2004 .02584 . \mathrm{x}$
Schauwecker, P.E., 2003. Genetic basis of kainateinduced excitotoxicity in mice: Phenotypic modulation of seizure-induced cell death. Epilepsy Res, 55: 201-210. DOI: 10.1016/S0920-1211(03)00115-3

Schoepp, D.D., 1994. Novel functions for subtypes of metabotropic glutamate receptors. Neurochem. Int., 24: 439-449. DOI: 10.1016/0197-0186(94)90092-2

Taketo, M., A.C. Schroeder, L.E. Mobraaten, K.B. Gunning and G. Hanten et al., 1991. FVB/N: An inbred mouse strain preferable for transgenic analyses. Proc. Natl. Acad. Sci. USA., 88: 2065-2069. DOI: $10.1073 /$ pnas.88.6.2065

Truong, D.D., R. Bhidayasiri and E. Wolters, 2008. Management of non-motor symptoms in advanced Parkinson disease. J. Neurol. Sci., 266: 216-228. DOI: 10.1016/j.jns.2007.08.015

Zhen-Hong, S., M. Xiang-Yang and Z. Rui-Liang, 2010. New advances in animal transgenic technology. Yi Chuan, 32: 539-547.

DOI: $10.3724 /$ SP.J.1005.2010.00539 